\title{
Analysis of $K^{+} \rightarrow e^{+} \nu_{e} \gamma$ in light-front quark model and chiral perturbation theory of order $p^{6}$
}

\author{
Chuan-Hung Chen ${ }^{1,2}$, Chao-Qiang Geng ${ }^{3}$ and Chong-Chung Lih ${ }^{4}$ \\ ${ }^{1}$ Department of Physics, National Cheng-Kung University, Tainan 701, Taiwan \\ ${ }^{2}$ National Center for Theoretical Sciences, Hsinchu 300, Taiwan \\ ${ }^{3}$ Department of Physics, National Tsing-Hua University, Hsinchu 300, Taiwan \\ ${ }^{4}$ General Education Center, Tzu-Chi College of Technology, Hualien 970, Taiwan
}

(Dated: September 11, 2021)

\begin{abstract}
Within the frameworks of the light-front quark model (LFQM) and chiral perturbation theory (ChPT) of $O\left(p^{6}\right)$, we reevaluate the form factors of the $K^{+} \rightarrow \gamma$ transition. We use these form factors to study the decay of $K^{+} \rightarrow e^{+} \nu_{e} \gamma$, which is dominated by the structure dependent contribution. We show the differential decay branching ratio as a function of $x=2 E_{\gamma} / m_{K}$, where $E_{\gamma}\left(m_{K}\right)$ is the photon energy (kaon mass). Explicitly, we find that, in the standard model with the cut of $x=0.01(0.1)$, the decay branching ratio of $K^{+} \rightarrow e^{+} \nu_{e} \gamma$ is $1.54(1.44) \times 10^{-5}$ and $1.57(1.47) \times 10^{-5}$ in the LFQM and ChPT, respectively.
\end{abstract}




\section{INTRODUCTION}

Experimentally, both decays of $K^{+} \rightarrow e^{+} \nu_{e}$ and $\mu^{+} \nu_{\mu}$ have been precisely measured with the decay branching ratios being $(1.55 \pm 0.05) \times 10^{-5}$ and $(63.44 \pm 0.14) \times 10^{-2}$ [1], respectively. The smallness of the electron mode can be easily understood as it is helicity suppressed with the suppression factor of $m_{e}^{2} / m_{\mu}^{2} \sim 2 \times 10^{-5}$ in comparison with the muon mode. For the corresponding radiative decays of $K^{+} \rightarrow \ell^{+} \nu_{\ell} \gamma(\ell=e, \mu)$, it is known that they receive two types of contributions: "inner bremsstrahlung" (IB) and "structure-dependent" (SD) [2, 3]. For the decay of $K^{+} \rightarrow e^{+} \nu_{e} \gamma$, while the IB contribution is still helicity suppressed and contains the electromagnetic coupling constant $\alpha$ as well, the SD part gives the dominant contribution to the decay rate as it is free of the helicity suppression. Similarly, the SD contribution is also important to the decay of $K^{+} \rightarrow \mu^{+} \nu_{\mu} \gamma[4]$.

In the standard model (SM), the decay amplitude of the SD part involves vector and axial-vector hadronic currents, which can be parametrized in terms of the vector form factor $F_{V}$ and axial-vector form factor $F_{A}$, respectively. However, the experimental determinations on these form factors are poorly given and model-dependent [5, 6, 7]. In particular, the experimental results on the decay rate of $K^{+} \rightarrow e^{+} \nu_{e} \gamma$ in Ref. [5, 6, 7] were based on the assumption of $F_{V}$ and $F_{A}$ being some constant values in the chiral perturbation theory (ChPT) at $O\left(p^{4}\right)[4]$. In the ongoing data analysis of the E949 experiment at BNL, more precision measurements on the decay of $K^{+} \rightarrow e^{+} \nu_{e} \gamma$ are expected [8] and thus, the modelindependent extractions of the SD form factors are possible. Theoretical calculations of $F_{V}$ and $F_{A}$ in the $K^{+} \rightarrow \gamma$ transition have been previously done in the ChPT at $O\left(p^{4}\right)$ [4] and $O\left(p^{6}\right)[9,10]$. However, the results of the ChPT at $O\left(p^{6}\right)$ [10] have not been fully applied to the decay of $K^{+} \rightarrow e^{+} \nu_{e} \gamma$ yet. Moreover, it is important if we could obtain information on $F_{V, A}$ in some QCD model other than the ChPT. For this purpose, in the present study we will also evaluate $F_{V, A}$ in the light front quark model (LFQM). We will use the form factors in both ChPT and LFQM to examine the decay of $K^{+} \rightarrow e^{+} \nu_{e} \gamma$.

This paper is organized as follows: We present the relevant formulas for the matrix elements and form factors for the decay of $K^{+} \rightarrow e^{+} \nu_{e} \gamma$ in Sec. II. In particular, we study the transition form factors of $K^{+} \rightarrow \gamma$ in the ChPT of $O\left(p^{6}\right)$ and LFQM. In Sec. III, we describe the differential decay rate of $K^{+} \rightarrow e^{+} \nu_{e} \gamma$. In Sec. IV, we show our numerical results on the form factors and the decay branching ratio in both ChPT and LFQM. We will 
also illustrate the differential decay branching ratio as a function of $x=2 E_{\gamma} / m_{K}$, where $E_{\gamma}$ and $m_{K}$ are the photon energy and kaon mass, respectively. We give our conclusions in Sec. V.

\section{MATRIX ELEMENTS AND FORM FACTORS}

In the SM, the amplitude of the decay $K^{+} \rightarrow e^{+} \nu_{e} \gamma\left(K_{e 2 \gamma}^{+}\right)$can be written in terms of IB and SD contributions, given by [3, 4, 11, 12]

$$
\begin{aligned}
M & =M_{I B}+M_{S D}, \\
M_{I B} & =i e \frac{G_{F}}{\sqrt{2}} \sin \theta_{c} F_{K} m_{e} \epsilon_{\alpha}^{*} K^{\alpha}, \\
M_{S D} & =-i e \frac{G_{F}}{\sqrt{2}} \sin \theta_{c} \epsilon_{\mu}^{*} L_{\nu} H^{\mu \nu},
\end{aligned}
$$

where

$$
\begin{aligned}
K^{\alpha} & =\bar{u}\left(p_{\nu}\right)\left(1+\gamma_{5}\right)\left(\frac{p_{K}^{\alpha}}{p_{K} \cdot q}-\frac{2 p_{e}^{\alpha}+\not q \gamma^{\alpha}}{2 p_{e} \cdot q}\right) v\left(p_{e}\right), \\
L_{\nu} & =\bar{u}\left(p_{\nu}\right) \gamma_{\nu}\left(1-\gamma_{5}\right) v\left(p_{e}\right), \\
H^{\mu \nu} & =\frac{F_{A}}{m_{K}}\left(-g^{\mu \nu} p_{K} \cdot q+p_{K}^{\mu} q^{\nu}\right)+i \frac{F_{V}}{m_{K}} \epsilon^{\mu \nu \alpha \beta} q_{\alpha} p_{K \beta},
\end{aligned}
$$

$\epsilon_{\alpha}$ is the photon polarization vector, $p_{K}, p_{\nu}, p_{e}$, and $q$ are the four-momenta of $K^{+}, \nu_{e}, e^{+}$, and $\gamma$, and $F_{K}$ and $F_{A(V)}$ are the $K$ meson decay constant and the axial-vector (vector) form factor corresponding to the axial-vector (vector) part of the weak currents, defined by

$$
\begin{aligned}
\left\langle 0\left|\bar{s} \gamma^{\mu} \gamma_{5} u\right| K^{+}\left(p_{K}\right)\right\rangle & =-i F_{K} p_{K}^{\mu}, \\
\left\langle\gamma(q)\left|\bar{u} \gamma^{\mu} \gamma_{5} s\right| K\left(p_{K}\right)\right\rangle & =e \frac{F_{A}}{m_{K}}\left[(p \cdot q) \epsilon^{* \mu}-\left(\epsilon^{*} \cdot p\right) q^{\mu}\right], \\
\left\langle\gamma(q)\left|\bar{u} \gamma^{\mu} s\right| K\left(p_{K}\right)\right\rangle & =i e \frac{F_{V}}{m_{K}} \varepsilon^{\mu \alpha \beta \nu} \epsilon_{* \alpha} q_{\beta} p_{\nu},
\end{aligned}
$$

respectively, with $p=p_{K}-q$ being the transfer momentum. We note that $\mathcal{M}_{I B}$ in Eq. (1) is suppressed due to the small electron mass $m_{e}$. In the decay of $K^{+} \rightarrow e^{+} \nu_{e} \gamma$, the form factors $F_{A, V}$ in Eq. (3) are the analytic functions of $p^{2}=\left(p_{K}-q\right)^{2}$ in the physical allowed region, given by

$$
m_{e}^{2} \leq p^{2} \leq m_{K}^{2}
$$

In the following discussion, we will first summarize the formulas for $F_{V, A}$ in the ChPT and then study these form factors in the LFQM. We note that similar calculations for the $P \rightarrow \gamma\left(P=K^{0}, D, B\right)$ transitions in the LFQM have been performed in Refs. [13, 14, 15]. 


\section{A. Chiral Perturbation Theory}

The chiral Lagrangians contain both normal and anomalous parts. At orders of $p^{m}$, the

non-anomalous and anomalous Lagrangians of $\mathcal{L}_{n}^{(m)}$ and $\mathcal{L}_{a}^{(m)}$ relevant to the $K_{e 2 \gamma}^{+}$decay are given by [10]

$$
\begin{gathered}
\mathcal{L}_{n}^{(2)}=\frac{F^{2}}{4} \operatorname{Tr}\left(D_{\mu} U D^{\mu} U^{\dagger}\right)+\frac{F^{2}}{4} \operatorname{Tr}\left(\chi U^{\dagger}+U \chi^{\dagger}\right), \\
\mathcal{L}_{n}^{(4)}=L_{1}\left[\operatorname{Tr}\left(D_{\mu} U D^{\mu} U^{\dagger}\right)\right]^{2}+L_{2} \operatorname{Tr}\left(D_{\mu} U D_{\nu} U^{\dagger}\right) \operatorname{Tr}\left(D^{\mu} U D^{\nu} U^{\dagger}\right) \\
+L_{3} \operatorname{Tr}\left(D_{\mu} U D^{\mu} U^{\dagger} D_{\nu} U D^{\nu} U^{\dagger}\right)+L_{4} \operatorname{Tr}\left(D_{\mu} U D^{\mu} U^{\dagger}\right) \operatorname{Tr}\left(\chi U^{\dagger}+U \chi^{\dagger}\right) \\
+L_{5} \operatorname{Tr}\left(D_{\mu} U D^{\mu} U^{\dagger}\left(\chi U^{\dagger}+U \chi^{\dagger}\right)\right)+L_{6}\left[\operatorname{Tr}\left(\chi U^{\dagger}+U \chi^{\dagger}\right)\right]^{2} \\
+L_{7}\left[\operatorname{Tr}\left(\chi^{\dagger} U-U^{\dagger} \chi\right)\right]^{2}+L_{8} \operatorname{Tr}\left(\chi U^{\dagger} \chi U^{\dagger}+U \chi^{\dagger} U \chi^{\dagger}\right) \\
+i L_{9} \operatorname{Tr}\left(L_{\mu \nu} D^{\mu} U D^{\nu} U^{\dagger}+R_{\mu \nu} D^{\mu} U^{\dagger} D^{\nu} U\right)+L_{10} \operatorname{Tr}\left(L_{\mu \nu} U R_{\mu \nu} U^{\dagger}\right) \\
\mathcal{L}_{n}^{(6)=} y_{17}\left\langle\chi_{+} h_{\mu \nu} h^{\mu \nu}\right\rangle+y_{18}\left\langle\chi_{+}\right\rangle\left\langle h_{\mu \nu} h^{\mu \nu}\right\rangle+y_{81}\left\langle\chi_{+} f_{+\mu \nu} f_{+}^{\mu \nu}\right\rangle \\
+y_{82}\left\langle\chi_{+}\right\rangle\left\langle f_{+\mu \nu} f_{+}^{\mu \nu}\right\rangle+i y_{83}\left\langle f_{+\mu \nu}\left\{\chi_{+}, u^{\mu} u^{\nu}\right\}\right\rangle+i y_{84}\left\langle\chi_{+}\right\rangle\left\langle f_{+\mu \nu} u^{\mu} u^{\nu}\right\rangle \\
+i y_{85}\left\langle f_{+\mu \nu} u^{\mu} \chi_{+} u^{\nu}\right\rangle+i y_{100}\left\langle f_{-\mu \nu}\left[f_{-}^{\nu \rho}, h_{\rho}^{\mu}\right]\right\rangle+y_{102}\left\langle\chi_{+} f_{-\mu \nu} f_{-}^{\mu \nu}\right\rangle \\
+y_{103}\left\langle\chi_{+}\right\rangle\left\langle f_{-\mu \nu} f_{-}^{\mu \nu}\right\rangle+y_{104}\left\langle f_{+\mu \nu}\left[f_{-}^{\mu \nu}, \chi_{-}\right]\right\rangle+y_{109}\left\langle\nabla_{\rho} f_{-\mu \nu} \nabla^{\rho} f_{-}^{\mu \nu}\right\rangle \\
+y_{110}\left\langle\nabla \rho f_{+\mu \nu}\left[h^{\nu \rho}, u^{\nu}\right]\right\rangle+\ldots .
\end{gathered}
$$

and [16, 17]

$$
\begin{aligned}
\mathcal{L}_{a}^{(4)}= & -\frac{1}{16 \pi^{2}} \epsilon^{\mu \nu \alpha \beta} \operatorname{Tr}\left(U \partial_{\mu} U^{+} \partial_{\nu} U \partial_{\alpha} U^{+} l_{\beta}-U^{+} \partial_{\mu} U \partial_{\nu} U^{+} \partial_{\alpha} U r_{\beta}\right) \\
& -\frac{i}{16 \pi^{2}} \epsilon^{\mu \nu \alpha \beta} \operatorname{Tr}\left(\partial_{\mu} U^{+} \partial_{\nu} l_{\alpha} U r_{\beta}-\partial_{\mu} U \partial_{\nu} r_{\alpha} U^{+} l_{\beta}\right) \\
& +U \partial_{\mu} U^{+}\left(l_{\nu} \partial_{\alpha} l_{\beta}+\partial_{\nu} l_{\alpha} l_{\beta}\right) \\
\mathcal{L}_{a}^{(6)}= & i C_{7} \epsilon^{\mu \nu \alpha \beta}\left\langle\chi_{-} f_{+\mu \nu} f_{+\alpha \beta}\right\rangle+i C_{11} \epsilon^{\mu \nu \alpha \beta}\left\langle\chi_{-}\left[f_{+\mu \nu}, f_{-\alpha \beta}\right]\right\rangle \\
+ & C_{22} \epsilon^{\mu \nu \alpha \beta}\left\langle u_{-}\left\{\nabla_{\gamma} f_{+\gamma \nu}, f_{+\alpha \beta}\right\}\right\rangle+\ldots,
\end{aligned}
$$

respectively, where $F$ is the meson decay constant in the chiral limit, $L_{i}, y_{j}$ and $C_{k}$ are unrenormalized coupling constants, $U$ is the unitary matrix, parametreized by

$$
U=\exp \left[i \frac{\sqrt{2}}{F}\left(\begin{array}{ccc}
\frac{\pi^{0}}{\sqrt{2}}+\frac{\eta}{\sqrt{6}} & \pi^{+} & K^{+} \\
\pi^{-} & -\frac{\pi^{0}}{\sqrt{2}}+\frac{\eta}{\sqrt{6}} & K^{0} \\
K^{-} & K^{0} & -\frac{2 \eta}{\sqrt{6}}
\end{array}\right)\right]
$$


$L_{\mu \nu}$ and $R_{\mu \nu}$ are the field-strength tensors of external sources, given by

$$
\begin{aligned}
& L_{\mu \nu}=\partial_{\mu} \ell_{\nu}-\partial_{\nu} \ell_{\mu}-i\left[\ell_{\mu}, \ell_{\nu}\right], \\
& R_{\mu \nu}=\partial_{\mu} r_{\nu}-\partial_{\nu} r_{\mu}-i\left[r_{\mu}, r_{\nu}\right],
\end{aligned}
$$

and the definitions of all other fields can be found in Ref. [10].

From the chiral Lagrangians in Eqs. (5) and (66), one obtains the tree and loop contributions to $F_{V}$ at $O\left(p^{6}\right)$ for the $K_{e 2 \gamma}^{+}$decay to be $[9,10]$

$$
\begin{aligned}
F_{V}\left(p^{2}\right) & =\frac{m_{K}}{4 \sqrt{2} \pi^{2} F_{K}}\left\{1-\frac{256}{3} \pi^{2} m_{K}^{2} C_{7}^{r}+256 \pi^{2}\left(m_{K}^{2}-m_{\pi}^{2}\right) C_{11}^{r}+\frac{64}{3} \pi^{2} p^{2} C_{22}^{r}\right. \\
& -\frac{1}{16 \pi^{2}\left(\sqrt{2} F_{K}\right)^{2}}\left[\frac{3}{2} m_{\eta}^{2} \ln \left(\frac{m_{\eta}^{2}}{\mu^{2}}\right)+\frac{7}{2} m_{\pi}^{2} \ln \left(\frac{m_{\pi}^{2}}{\mu^{2}}\right)+3 m_{K}^{2} \ln \left(\frac{m_{K}^{2}}{\mu^{2}}\right)\right. \\
& -2 \int\left[x m_{\pi}^{2}+(1-x) m_{K}^{2}-x(1-x) p^{2}\right] \ln \left(\frac{x m_{\pi}^{2}+(1-x) m_{K}^{2}-x(1-x) p^{2}}{\mu^{2}}\right) d x \\
& -2 \int\left[x m_{\eta}^{2}+(1-x) m_{K}^{2}-x(1-x) p^{2}\right] \ln \left(\frac{x m_{\eta}^{2}+(1-x) m_{K}^{2}-x(1-x) p^{2}}{\mu^{2}}\right) d x \\
& \left.\left.-4 \int m_{\pi}^{2} \ln \left(\frac{m_{\pi}^{2}}{\mu^{2}}\right) d x\right]\right\},
\end{aligned}
$$

where the wave function and decay constant renormalizations have been included and $C_{i}^{r}$ are the renormalized coefficients. From Eq. (5), the tree and loop contributions to $F_{A}$ of $O\left(p^{6}\right)$ lead to [10]

$$
\begin{aligned}
F_{A}\left(p^{2}\right)= & \frac{4 \sqrt{2} m_{K}}{F_{K}}\left(L_{9}^{r}+L_{10}^{r}\right)+\frac{m_{K}}{6 F_{K}^{3}(2 \pi)^{8}}\left[142.65\left(m_{K}^{2}-p^{2}\right)-198.3\right] \\
- & \frac{m_{K}}{4 \sqrt{2} F_{K}^{3} \pi^{2}}\left\{\left(4 L_{3}^{r}+7 L_{9}^{r}+7 L_{10}^{r}\right) m_{\pi}^{2} \ln \left(\frac{m_{\pi}^{2}}{m_{\rho}^{2}}\right)+3\left(L_{9}^{r}+L_{10}^{r}\right) m_{\eta}^{2} \ln \left(\frac{m_{\eta}^{2}}{m_{\rho}^{2}}\right)\right. \\
& \left.+2\left(8 L_{1}^{r}-4 L_{2}^{r}+4 L_{3}^{r}+7 L_{9}^{r}+7 L_{10}^{r}\right) m_{K}^{2} \ln \left(\frac{m_{K}^{2}}{m_{\rho}^{2}}\right)\right\} \\
- & \frac{4 \sqrt{2} m_{K}}{3 F_{K}^{3}}\left\{2 m_{\pi}^{2}\left(18 y_{18}^{r}-2 y_{81}^{r}-6 y_{82}^{r}+2 y_{83}^{r}+3 y_{84}^{r}-y_{85}^{r}+6 y_{103}^{r}\right)\right. \\
& +2 m_{K}^{2}\left(18 y_{17}^{r}+36 y_{18}^{r}-4 y_{81}^{r}-12 y_{82}^{r}+4 y_{83}^{r}+6 y_{84}^{r}+4 y_{85}^{r}-3 y_{100}^{r}\right. \\
& \left.\left.+6 y_{102}^{r}+12 y_{103}^{r}-6 y_{104}^{r}+3 y_{109}^{r}\right)+\frac{3}{2}\left(m_{K}^{2}-p^{2}\right)\left(2 y_{100}^{r}-4 y_{109}^{r}+y_{110}^{r}\right)\right\},
\end{aligned}
$$

where $L_{i}^{r}$ and $y_{i}^{r}$ are the renormalized coupling constants. Note that the first terms in Eqs. (9) and (10) correspond to $F_{V}$ and $F_{A}$ at $O\left(p^{4}\right)$ [4, 18], respectively. We remark that the expressions of Eqs. (9) and (10) have not been explicitly shown in the literature [9, 10]. 


\section{B. Light Front Quark Model}

In the framework of the LFQM [13, 14, 15], the physical accessible kinematics region is $0 \leq p^{2} \leq M_{K}^{2}$ due to the time-like momentum transfers. The general structure of the phenomenological light front (LF) meson wave function is based only on the $q \bar{q}$ Fock space sector. It can be expressed by an anti-quark $\bar{s}$ and a quark $u$ with the total momentum $(p+q)$ such as:

$$
\begin{aligned}
|K(p+q)\rangle=\sum_{\lambda_{1} \lambda_{2}} \int[ & \left.d k_{1}\right]\left[d k_{2}\right] 2(2 \pi)^{3} \delta^{3}\left(p+q-k_{1}-k_{2}\right) \\
& \times \Phi_{K}^{\lambda_{1} \lambda_{2}}\left(z, k_{\perp}\right) b_{\bar{s}}^{+}\left(k_{1}, \lambda_{1}\right) d_{u}^{+}\left(k_{2}, \lambda_{2}\right)|0\rangle,
\end{aligned}
$$

where $\Phi_{K}^{\lambda_{1} \lambda_{2}}$ is the amplitude of the corresponding $\bar{s}(u)$ and $k_{1(2)}$ is the on-mass shell LF momentum of the internal quark. The LF relative momentum variables $\left(z, k_{\perp}\right)$ are defined by

$$
\begin{aligned}
& k_{1}^{+}=z_{1}(p+q)^{+}, \quad k_{2}^{+}=z_{2}(p+q)^{+}, \quad z_{1}+z_{2}=1, \\
& k_{1 \perp}=z_{1}(p+q)_{\perp}+k_{\perp}, \quad k_{2 \perp}=z_{2}(p+q)_{\perp}-k_{\perp},
\end{aligned}
$$

and

$$
\Phi_{K}^{\lambda_{1} \lambda_{2}}\left(z, k_{\perp}\right)=\left(\frac{k_{1}^{+} k_{2}^{+}}{2\left[M_{0}^{2}-\left(m_{s}-m_{u}\right)^{2}\right]}\right)^{\frac{1}{2}} \bar{u}\left(k_{1}, \lambda_{1}\right) \gamma^{5} v\left(k_{2}, \lambda_{2}\right) \phi\left(z, k_{\perp}\right),
$$

with $\phi\left(z, k_{\perp}\right)$ being the space part of the wave function, which depends on the dynamics. The amplitude of $\phi\left(z, k_{\perp}\right)$ can be solved in principles by the LF QCD bound state equation [19, 20]. However, we use the Gaussian type wave function in this study:

$$
\phi\left(z, k_{\perp}\right)=N \sqrt{\frac{d k_{z}}{d z}} \exp \left(-\frac{\vec{k}^{2}}{2 \omega_{K}^{2}}\right) .
$$

From Eqs. (11)-(14), the hadronic matrix elements in Eq. (3) are found to be

$$
\begin{aligned}
& \left\langle\gamma(q)\left|\bar{s} \gamma^{\mu}\left(1-\gamma_{5}\right) u\right| K(p+q)\right\rangle=\int \frac{d^{4} k_{1}^{\prime}}{(2 \pi)^{4}} \Lambda_{K} \\
& \times\left\{\gamma_{5} \frac{i\left(-k_{2}^{\prime}+m_{u}\right)}{k_{2}^{\prime 2}-m_{u}^{2}+i \epsilon} i e_{u} \phi \frac{i\left(k_{1}+m_{u}\right)}{k_{1}^{2}-m_{u}^{2}+i \epsilon} \gamma^{\mu}\left(1-\gamma_{5}\right) \frac{i\left(k_{1}^{\prime}+m_{s}\right)}{k_{1}^{\prime 2}-m_{s}^{2}+i \epsilon}\right. \\
& \left.+\left(u \leftrightarrow s, k_{1}^{\prime}\left(k_{1}\right) \leftrightarrow k_{2}^{\prime}\left(k_{2}\right)\right)\right\},
\end{aligned}
$$


where $\Lambda_{K}$ is a vertex function related to the $u \bar{s}$ bound state of the $K$ meson, $k_{2}=q-k_{1}$ and $k_{1}^{\prime}=(p+q)-k_{2}^{\prime}=k_{1}+p$. After integrating over the LF momentum $k_{1}^{-}$in Eq. (15), we get

$$
\begin{aligned}
& \left\langle\gamma(q)\left|\bar{s} \gamma^{\mu}\left(1-\gamma_{5}\right) u\right| K(p+q)\right\rangle \\
& =\int_{q}^{p+q}\left[d^{3} k_{1}^{\prime}\right]\left\{\frac{1}{k_{1}^{-}-k_{1 o n}^{-}}\left(\left.I^{\mu \nu}\right|_{k_{1 o n}^{\prime}-}\right) \frac{\Lambda_{P}}{k_{2}^{\prime-}-k_{2 o n}^{\prime-}}+\left(u \leftrightarrow s, k_{1}^{\prime}\left(k_{1}\right) \leftrightarrow k_{2}^{\prime}\left(k_{2}\right)\right)\right\},
\end{aligned}
$$

where

$$
\begin{aligned}
& {\left[d^{3} k_{1}^{\prime}\right]=\frac{d k_{1}^{+} d k_{1 \perp}}{2(2 \pi)^{3} k_{1}^{\prime+} k_{2}^{\prime+} k_{1}^{+}},} \\
& \left.\left.I^{\mu \nu}\right|_{k_{1 o n}^{-}}=\operatorname{Tr}\left\{\gamma_{5}\left(-k_{2}^{\prime}+m_{u}\right) i e_{u} \phi_{\left(k_{1}\right.}+m_{u}\right) \gamma^{\mu}\left(1-\gamma_{5}\right)\left(k_{1}^{\prime}+m_{s}\right)\right\}, \\
& k_{\text {ion }}^{-}=\frac{m_{i}^{2}+k_{i \perp}^{2}}{k_{i}^{+}}, k_{1(2)}^{\prime}=p_{\text {on }}^{-}-k_{2(1) o n}^{\prime}, k_{1}^{-}=q^{-}-k_{2 o n}^{-},
\end{aligned}
$$

with $\{$ on $\}$ representing the on-shell particles. For the kaon, the vertex function $\Lambda_{P}$ in Eqs. (15) and (16) is given by [21, 22]:

$$
\frac{\Lambda_{P}}{k_{2}^{\prime-}-k_{2 o n}^{\prime-}} \rightarrow \frac{\sqrt{k_{1}^{\prime+} k_{2}^{\prime+}}}{\sqrt{2} \widetilde{M}_{0}} \phi\left(z^{\prime}, k_{\perp}\right) \text {. }
$$

To calculate the right hand part of Eq. (16), we choose a frame with the transverse momentum $p_{\perp}=0$ so that $p^{2}=p^{+} p^{-} \geq 0$ covers the entire range of the momentum transfers. Here, we have used the LF momentum variables $\left(x, k_{\perp}\right)$. Hence, the relevant quark momentum variables in Fig. 1 are

$$
\begin{gathered}
k_{1}^{\prime+}=\left(1-z^{\prime}\right)(p+q)^{+}, k_{2}^{\prime+}=z^{\prime}(p+q)^{+}, k_{1 \perp}^{\prime}=\left(1-z^{\prime}\right) q_{\perp}+k_{\perp}^{\prime}, k_{2 \perp}^{\prime}=z^{\prime} q_{\perp}-k_{\perp}^{\prime}, \\
k_{1}^{+}=(1-z) q^{+}, \quad k_{2}^{+}=z q^{+}, \quad k_{1 \perp}=(1-z) q_{\perp}+k_{\perp}, \quad k_{2 \perp}=z q_{\perp}-k_{\perp} .
\end{gathered}
$$

By considering the good component as " $\mu=+$ ", the hadronic matrix elements in Eq. (3) can be rewritten as:

$$
\begin{aligned}
\left\langle\gamma(q)\left|s_{+}^{+} \gamma_{5} u_{+}\right| K(p+q)\right\rangle & =-e \frac{F_{A}}{2 m_{K}}\left(\epsilon_{\perp}^{*} \cdot q_{\perp}\right) p^{+} \\
\left\langle\gamma(q)\left|s_{+}^{+} u_{+}\right| K(p+q)\right\rangle & =-i e \frac{F_{V}}{2 m_{K}} \epsilon^{i j} \epsilon_{i}^{*} q_{j} p^{+} .
\end{aligned}
$$

Using Eq, (19), the trace part $I^{\mu \nu}$ in Eq. (17) can be carried out. By comparing Eq. (16) 
with Eq. (20), we obtain the form factors $F_{V, A}$ to be:

$$
\begin{aligned}
F_{A}\left(p^{2}\right)=4 m_{K} \int & \frac{d z d^{2} k_{\perp}}{2(2 \pi)^{3}} \Phi\left(z^{\prime}, k_{\perp}^{2}\right) \frac{1}{1-z^{\prime}} \\
& \times\left\{\frac{2}{3} \frac{m_{u}-A k_{\perp}^{2} \Theta}{m_{u}^{2}+k_{\perp}^{2}}+\frac{1}{3} \frac{m_{s}+B k_{\perp}^{2} \Theta}{m_{s}^{2}+k_{\perp}^{2}}\right\}, \\
F_{V}\left(p^{2}\right)= & 8 m_{K} \int \frac{d z d^{2} k_{\perp}}{2(2 \pi)^{3}} \Phi\left(z^{\prime}, k_{\perp}^{2}\right) \frac{1}{1-z^{\prime}} \\
& \left\{\frac{2}{3} \frac{m_{u}-z^{\prime}\left(m_{s}-m_{u}\right) k_{\perp}^{2} \Theta}{m_{u}^{2}+k_{\perp}^{2}}-\frac{1}{3} \frac{m_{s}+\left(1-z^{\prime}\right)\left(m_{s}-m_{u}\right) k_{\perp}^{2} \Theta}{m_{s}^{2}+k_{\perp}^{2}}\right\},
\end{aligned}
$$

where

$$
\begin{aligned}
A & =(1-2 z) z^{\prime}\left(m_{s}-m_{u}\right)-2 z m_{u} \\
B & =(1-2 z) z^{\prime} m_{s}+m_{s}+(1-2 z)\left(1-z^{\prime}\right) m_{u} \\
\Phi\left(z, k_{\perp}^{2}\right) & =N\left(\frac{z(1-z)}{2\left[M_{0}^{2}-\left(m_{s}-m_{u}\right)^{2}\right]}\right)^{1 / 2} \sqrt{\frac{d k_{z}}{d z}} \exp \left(-\frac{\vec{k}^{2}}{2 \omega_{K}^{2}}\right), \\
\Theta & =\frac{1}{\Phi\left(z, k_{\perp}^{2}\right)} \frac{d \Phi\left(z, k_{\perp}^{2}\right)}{d k_{\perp}^{2}} \\
z^{\prime} & =z\left(1-\frac{p^{2}}{M_{K}^{2}}\right), \vec{k}=\left(\vec{k}_{\perp}, \vec{k}_{z}\right), \\
N & =4\left(\frac{\pi}{\omega_{K}^{2}}\right)^{\frac{3}{4}}, \quad k_{z}=\left(z-\frac{1}{2}\right) M_{0}+\frac{m_{s}^{2}-m_{u}^{2}}{2 M_{0}} \\
M_{0}^{2} & =\frac{k_{\perp}^{2}+m_{u}^{2}}{z}+\frac{k_{\perp}^{2}+m_{s}^{2}}{1-z} .
\end{aligned}
$$

\section{DIFFERENTIAL DECAY RATE}

In the $K^{+}$rest frame, the partial decay rate for $K^{+} \rightarrow e^{+} \nu_{e} \gamma$ is given by [1]

$$
d \Gamma=\frac{1}{(2 \pi)^{3}} \frac{1}{8 m_{K}}|M|^{2} d E_{\gamma} d E_{e},
$$

where $E_{\gamma}$ and $E_{e}$ are photon and electron energies, respectively. To describe the kinematics of $K^{+} \rightarrow e^{+} \nu_{e} \gamma$, we introduce two dimensionless variables, defined by $x=2 E_{\gamma} / m_{K}$ and $y=2 E_{e} / m_{K}$, with their physical allowed regions being

$$
\begin{aligned}
0 & \leq x \leq 1-r_{e} \\
1-x+\frac{r_{e}}{1-x} & \leq y \leq 1+r_{e}
\end{aligned}
$$


where $r_{e}=m_{e}^{2} / m_{K}^{2}$. The relation between the transfer momentum $p^{2}$ and $x$ is given by:

$$
p^{2}=m_{K}^{2}(1-x)
$$

From Eqs . (1) and (23), we obtain the double differential decay rate of $K^{+} \rightarrow e^{+} \nu_{e} \gamma$ as

$$
\frac{d^{2} \Gamma}{d x d y}=\frac{m_{K}^{5}}{64 \pi^{2}} \alpha G_{F}^{2} \sin ^{2} \theta_{c}(1-\lambda) A(x, y)
$$

where $\lambda=\left(x+y-1-r_{e}\right) / x$ and

$$
\begin{aligned}
A(x, y)=A_{I B}(x, y)+ & A_{S D^{+}}(x, y)+A_{S D^{-}}(x, y)+A_{I N T^{+}}(x, y)+A_{I N T^{-}}(x, y), \\
A_{I B}(x, y) & =\frac{4 r_{e}\left|F_{K}\right|^{2}}{m_{K}^{2} \lambda x^{2}}\left[x^{2}+2\left(1-r_{e}\right)\left(1-x-\frac{r_{e}}{\lambda}\right)\right], \\
A_{S D^{+}}(x, y) & =\left|F_{V}+F_{A}\right|^{2} \frac{x^{2} \lambda^{2}}{1-\lambda}\left(1-x-\frac{r_{e}}{\lambda}\right), \\
A_{S D^{-}}(x, y) & =\left|F_{V}-F_{A}\right|^{2} x^{2}(y-\lambda), \\
A_{I N T^{+}}(x, y) & =-\frac{4 r_{e}}{m_{K}} \operatorname{Re}\left[F_{K}\left(F_{V}+F_{A}\right)^{*}\right]\left(1-x-\frac{r_{e}}{\lambda}\right), \\
A_{I N T^{-}}(x, y) & =\frac{4 r_{e}}{m_{K}} \operatorname{Re}\left[F_{K}\left(F_{V}-F_{A}\right)^{*}\right] \frac{1-y+\lambda}{\lambda} .
\end{aligned}
$$

By integrating out the $y$ variable in Eq. (26), we obtain the differential decay rate as a function of $x$ to be

$$
\frac{d \Gamma}{d x}=\frac{m_{K}^{5}}{64 \pi^{2}} \alpha G_{F}^{2} \sin ^{2} \theta_{c} A(x)
$$

where

$$
\begin{aligned}
& A(x)=A_{I B}(x)+A_{S D^{+}}(x)+A_{S D^{-}}(x)+A_{I N T^{+}}(x)+A_{I N T^{-}}(x), \\
& A_{I B}(x)=\frac{4 r_{e} F_{K}^{2}}{m_{K}^{2}}\left[\frac{\left(x+r_{e}-1\right)\left[x^{2}+4\left(1-r_{e}\right)(1-x)\right]}{1-x}\right. \\
& \left.-\frac{x^{2}+2\left(1-r_{e}\right)\left(1-x+r_{e}\right)}{x} \ln \frac{r_{e}}{1-x}\right] \text {, } \\
& A_{S D^{+}}(x)=\left|F_{V}+F_{A}\right|^{2} x^{3}\left[\frac{1-x}{3}-\frac{r_{e}}{2}+\frac{r_{e}^{3}}{6(1-x)^{2}}\right] \text {, } \\
& A_{S D^{-}}(x)=\left|F_{V}-F_{A}\right|^{2} x^{3}\left[\frac{1-x}{3}-\frac{r_{e}}{2}+\frac{r_{e}^{3}}{6(1-x)^{2}}\right] \text {, } \\
& A_{I N T^{+}}(x)=\frac{4 r_{e}}{m_{K}} \operatorname{Re}\left[F_{K}\left(F_{V}+F_{A}\right)^{*}\right] x\left[\frac{1-x}{2}-\frac{r_{e}^{2}}{2(1-x)}+r_{e} \ln \frac{r_{e}}{1-x}\right] \text {, } \\
& A_{I N T^{-}}(x)=\frac{4 r_{e}}{m_{K}} \operatorname{Re}\left[F_{K}\left(F_{V}-F_{A}\right)^{*}\right] x\left[\frac{-1+3 x}{2}+\frac{r_{e}^{2}-2 x r_{e}}{2(1-x)}+\left(x-r_{e}\right) \ln \frac{r_{e}}{1-x}\right]
\end{aligned}
$$

It is clear that the contributions to the decay rate from the $I B$ and $I N T^{ \pm}$parts are suppressed due to the small electron mass. 


\section{NUMERICAL RESULTS}

The numerical values of $F_{A, V}\left(p^{2}\right)$ in the ChPT of $O\left(p^{6}\right)$ have been shown in Figs. 5 and 6 of Ref. [10]. To compare these values with those in the LFQM, we plot the results in Figs. 1 and 2. In these figures, we have also included the results in the ChPT at $O\left(p^{4}\right)$. For the

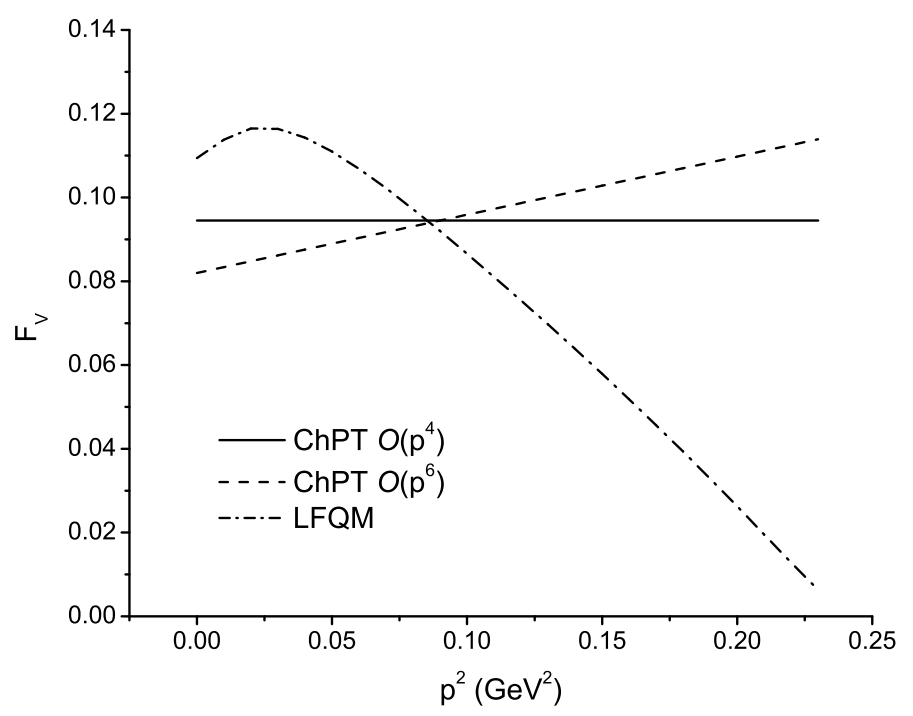

FIG. 1: $F_{V}\left(p^{2}\right)$ as a function of the transfer momentum $p^{2}$.

calculations of the ChPT [10], we have taken $m_{K}=0.495, m_{\pi}=0.14, m_{\eta}=0.55$ and $m_{\rho}=$ $0.77, F_{K}=0.112 \mathrm{GeV}$ and the renormalized coefficients of $\left(L_{1}^{r}, L_{2}^{r}, L_{3}^{r}, L_{9}^{r}, L_{10}^{r}\right),\left(C_{7}^{r}, C_{11}^{r}, C_{22}^{r}\right)$ and $\left(y_{100}^{r}, y_{10 \Lambda}^{r}, y_{109}^{r}, y_{110}^{r}\right)$ to be $(0.53,0.71,-2.72,6.9,-5.5) \times 10^{-3}[23],(0.013,-6.37,6.52) \times$ $10^{-3} \mathrm{GeV}^{-2}$ [24] and $(1.09,-0.36,0.40,-0.52) \times 10^{-4} / F_{K}^{2}$ [25], respectively. For some other possible sets of coefficients, see Ref. [10] as well as the recent review in Ref. [26]. We note that we have ignored the contributions from $p^{2}$-nondependent terms involving $y_{i}^{r}$. On the other hand, the $p^{2}$-dependence of $F_{A}\left(p^{2}\right)$ for the ChPT at $O\left(p^{6}\right)$ are insensitive due to the small contributions related to $y_{i}^{r}[10]$. We emphasize that as illustrated in Figs. 1 and 2 , the form factors $F_{V, A}$ at $O\left(p^{4}\right)$ in the ChPT are constants [4]. To evaluate the form factors of $F_{V, A}$ from Eq. (21) in the LFQM, we have used $m_{u}=0.26, m_{s}=0.37$ and $\omega_{K}=0.382$ in $G e V$. In Table @, we explicitly display the values of $F_{V, A}\left(p^{2}=0\right)$.

By integrating out the variable $x$ in Eq. (28), in Table \ we give the decay branching ratio of $K^{+} \rightarrow e^{+} \nu_{e} \gamma$ in (a) the ChPT at $O\left(p^{4}\right)$, (b) the ChPT of $O\left(p^{6}\right)$ and (c) the LFQM. 


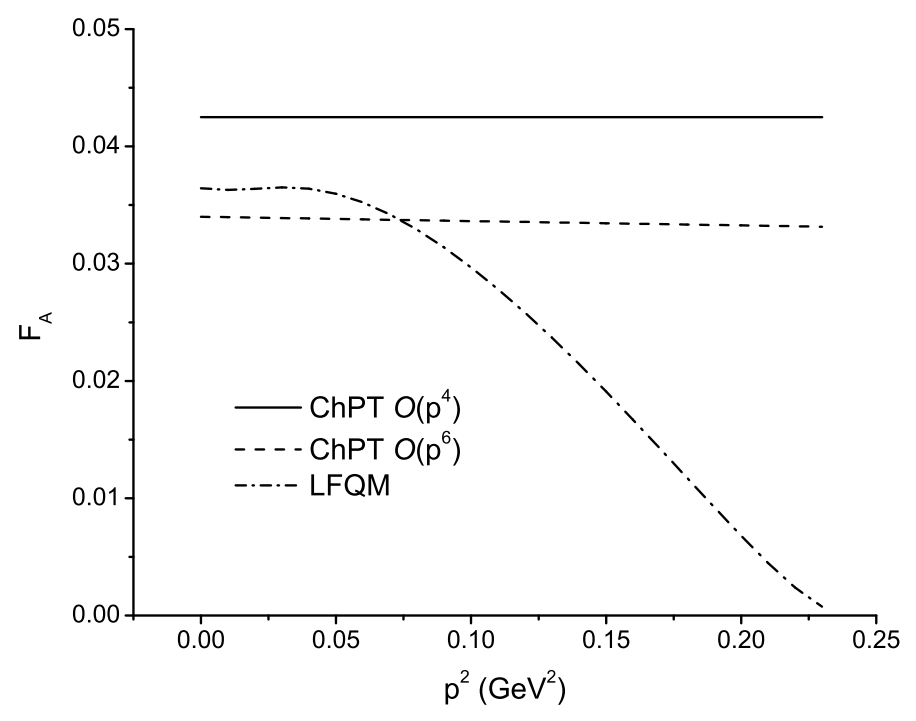

FIG. 2: $\quad F_{A}\left(p^{2}\right)$ as a function of the transfer momentum $p^{2}$.

TABLE I: The form factors of $F_{V}(0)$ and $F_{A}(0)$ in (a) the ChPT at $O\left(p^{4}\right)$ [4], (b) the ChPT of $O\left(p^{6}\right)$ and (c) the LFQM.

\begin{tabular}{|c||c|c|}
\hline Model & $F_{V}(0)$ & $F_{A}(0)$ \\
\hline \hline$(\mathrm{a})$ & 0.0945 & 0.0425 \\
\hline$(\mathrm{b})$ & 0.082 & 0.034 \\
\hline$(\mathrm{c})$ & 0.106 & 0.036 \\
\hline
\end{tabular}

Here, as the IB term diverges at the limit of $x \rightarrow 0$ corresponding to $p^{2} \rightarrow p_{\max }^{2}=m_{K}^{2}$, we have used the cuts of $x=0.01$ and 0.1 , respectively. With the cuts, from Table II we see that both IB and $\mathrm{INT}^{ \pm}$contributions are much smaller than the $\mathrm{SD}^{ \pm}$ones, which are insensitive to the cut. We remarks that in Table II, our results for the $\mathrm{SD}^{+}$contribution to the decay branching ratio in the ChPT of $O\left(p^{6}\right)$ and LFQM are 1.15 and $1.12 \times 10^{-5}$, which are smaller than that of $1.52 \pm 0.23 \times 10^{-5}$ [5, 6] quoted by the PDG [1], respectively. Note that the value in the PDG was based on the combination of the data in Refs. [5] and [6], in which large constant values of $F_{A}+F_{V}=0.150_{-0.023}^{+0.018}$ and $0.147 \pm 0.011$ were used, respectively. It is clear that to compare the data with the theoretical predictions, proper form factors should be used in the data analysis. 
To show the behaves of the various contributions in the ChPT and LFQM, we present the $\mathrm{IB}$ and $\mathrm{SD}^{ \pm}$parts of the differential decay branching ratio as functions of $x$ in Fig. 3 . Here, we do not plot the INT $^{ \pm}$contributions in Fig. 4 as they are vanishingly small. As shown in the figure, in the small $x$ region there is an enhancement for the IB part, whereas those from the $\mathrm{SD}^{ \pm}$parts are close to zero. In Fig. 4, we also display the spectrum of the differential decay branching ratio vs. $x$ in the ChPT at both $O\left(p^{4}\right)$ and $O\left(p^{6}\right)$ and the LFQM.

TABLE II: The decay branching ratio of $K^{+} \rightarrow e^{+} \nu_{e} \gamma\left(\right.$ in units of $10^{-5}$ ) in (a) the ChPT at $O\left(p^{4}\right)$, (b) the ChPT of $O\left(p^{6}\right)$ and (c) the LFQM with the cuts of $x=0.01$ and $x=0.1$, respectively.

\begin{tabular}{|c|c||c|c|c|c|c|c|}
\hline Model & Cut & $\mathrm{IB}$ & $\mathrm{SD}^{+}$ & $\mathrm{SD}^{-}$ & $\mathrm{INT}^{+}$ & $\mathrm{INT}^{-}$ & Total \\
\hline \hline$(\mathrm{a})$ & $x=0.01$ & $1.65 \times 10^{-1}$ & 1.34 & $1.93 \times 10^{-1}$ & $6.43 \times 10^{-5}$ & $-1.10 \times 10^{-3}$ & 1.70 \\
& $x=0.1$ & $0.69 \times 10^{-1}$ & 1.34 & $1.93 \times 10^{-1}$ & $6.43 \times 10^{-5}$ & $-1.10 \times 10^{-3}$ & 1.60 \\
\hline (b) & $x=0.01$ & $1.65 \times 10^{-1}$ & 1.15 & $2.58 \times 10^{-1}$ & $6.22 \times 10^{-5}$ & $-1.21 \times 10^{-3}$ & 1.57 \\
& $x=0.1$ & $0.69 \times 10^{-1}$ & 1.15 & $2.58 \times 10^{-1}$ & $6.22 \times 10^{-5}$ & $-1.21 \times 10^{-3}$ & 1.47 \\
\hline (c) & $x=0.01$ & $1.65 \times 10^{-1}$ & 1.12 & $2.59 \times 10^{-1}$ & $4.33 \times 10^{-5}$ & $-1.29 \times 10^{-3}$ & 1.54 \\
& $x=0.1$ & $0.69 \times 10^{-1}$ & 1.12 & $2.59 \times 10^{-1}$ & $4.33 \times 10^{-5}$ & $-1.29 \times 10^{-3}$ & 1.44 \\
\hline
\end{tabular}

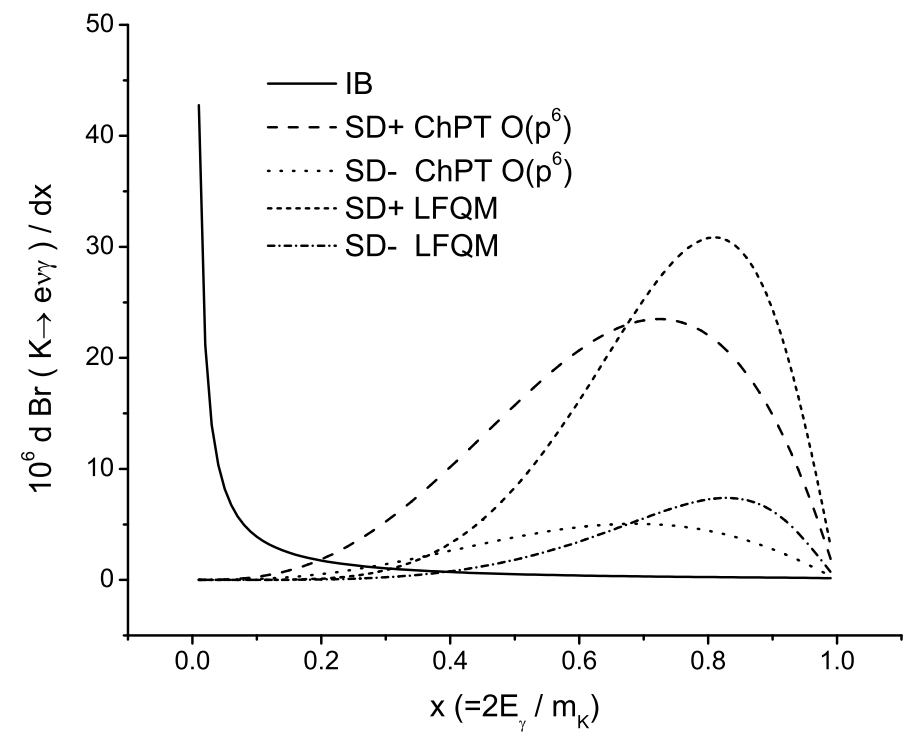

FIG. 3: The IB and $\mathrm{SD}^{ \pm}$parts of the differential decay branching ratio as functions of $x=2 E_{\gamma} / m_{K}$. 


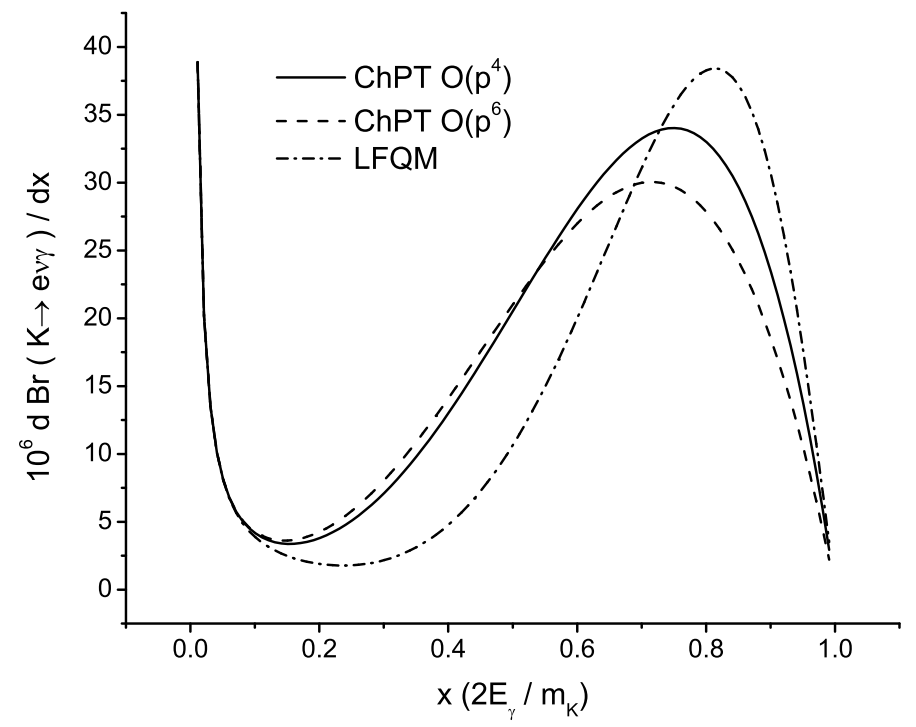

FIG. 4: The differential decay branching ratio as a function of $x=2 E_{\gamma} / m_{K}$.

From Fig. 4, we see that in the region of $x<0.7$ or $E_{\gamma}<173 \mathrm{MeV}$, the decay branching ratio in the LFQM is much smaller than that in the ChPT at $O\left(p^{6}\right)$. On the other hand, in the region of $x>0.7$ the statement is reversed. However, if we only consider the contributions in the ChPT at $O\left(p^{4}\right)$, the conclusion is weaker. In Table III, we illustrate the decay branching ratio in the regions of $0.1<x<0.7$ and $0.7<x<1$ from the various approaches, respectively. The main reasons for the differences are due to the form factors. The form

TABLE III: The decay branching ratio of $K^{+} \rightarrow e^{+} \nu_{e} \gamma$ (in units of $10^{-5}$ ) in the regions of $0.1<x<0.7$ and $0.7<x<1$ from the various approaches, respectively.

\begin{tabular}{|c||c|c|c|}
\hline Region & ChPT of $O\left(p^{4}\right)$ & ChPT of $O\left(p^{6}\right)$ & LFQM \\
\hline \hline $0.1<x<0.7$ & 0.871 & 0.871 & 0.541 \\
\hline $0.7<x<1$ & 0.733 & 0.606 & 0.902 \\
\hline
\end{tabular}

factors of the ChPT at $O\left(p^{4}\right)$ are constant and straight lines at $O\left(p^{6}\right)$, whereas in the LFQM they are the overlap between the wave functions of the $K$ meson and photon and become zero when $x \rightarrow 0$ or $p^{2} \rightarrow p_{\max }^{2}=m_{K}^{2}$. It is clear in the future data analysis such as the one at the experiment BNL-E949 [8], one could concentrate on these two regions to find out which model is preferred. 


\section{CONCLUSIONS}

We have studied the axial-vector and vector form factors of the $K^{+} \rightarrow \gamma$ transition in the LFQM and ChPT of $O\left(p^{6}\right)$. Based on these form factors, we have calculated the decay branching ratio of $K^{+} \rightarrow e^{+} \nu_{e} \gamma$. We have demonstrated that the SD part gives the dominant contribution to the decay in the whole allowed region of the photon energy except the low endpoint. Explicitly, we have found that, in the SM with the cut of $x=0.01(0.1)$, the decay branching ratio of $K^{+} \rightarrow e^{+} \nu_{e} \gamma$ is $1.54(1.44) \times 10^{-5}$ and $1.57(1.47) \times 10^{-5}$ in the LFQM and ChPT, respectively. Future precision experimental measurements on the decay

spectrum [8] should give us some useful information to determine the SD contribution as well as the vector and axial-vector form factors.

\section{Acknowledgments}

We would like to thank Professor D. A. Bryman for the suggestion of studying the decay spectrum and many useful discussions. This work is supported in part by the National Science Council of R.O.C. under Contract \#s: NSC-95-2112-M-006-013-MY2, NSC-95-2112M-007-059-MY3 and NSC-95-2112-M-277-001.

[1] Particle Data Group, W.M. Yao et al., J. Phys. G33, 1 (2006).

[2] J. T. Goldman and W. J. Wilson, Phys. Rev. D 15, 709 (1977), and references therein.

[3] D. A. Bryman et al., Phys. Rep. 88, 151 (1982).

[4] J. Bijnens, G.Ecker, and J. Gasser, Nucl. Phys. B 396, 81 (1993).

[5] K. S. Heard et al., Phys. Lett. B 55, 324 (1975).

[6] J. Heintze et al., Nucl. Phys. B 149, 365 (1979).

[7] S. Adler et al. [E787 Collaboration], Phys. Rev. Lett. 85, 2256 (2000); see also M. R. Convery, Ph.D Thesis, PRINCETON/HEP/96-11.

[8] D. A. Bryman, private communications.

[9] L. Ametller, J. Bijnens, A. Braman and F. Cornet, Phys. Lett. B303, 140 (1993).

[10] C. Q. Geng, I. L. Ho and T. H. Wu, Nucl. Phys. B684, 281 (2004).

[11] C. Q. Geng and S. K. Lee, Phys. Rev. D51, 99 (1995). 
[12] C. H. Chen, C. Q. Geng and C. C. Lih, Phys. Rev. D 56, 6856 (1997).

[13] C. Q. Geng, C. C. Lih and W. M. Zhang, Phys. Rev. D 57, 5697 (1998).

[14] C. C. Lih, C. Q. Geng and W. M. Zhang, Phys. Rev. D 59, 114002 (1999); C. Q. Geng, C. C. Lih and W. M. Zhang, Phys. Rev. D 62, 074017 (2000); Mod. Phys. Lett. A 15, 2087 (2000).

[15] C. Q. Geng, C. C. Lih and C. C. Liu, Phys. Rev. D62, 034019 (2000).

[16] A. Dobado, M. J. Herrero and T. N. Truong, Phys. Lett. B235, 134 (1990).

[17] Olof Strandberg, hep-ph/0302064

[18] J. F. Donoghue and B. R. Holstein, Phys. Rev. D40, 2378 (1989); Phys. Rev. D40, 3700 (1989).

[19] W. M. Zhang and A. Harindranath, Phys. Rev. D48, 4881 (1993).

[20] K. G. Wilson et al., Phys.Rev. D49, 6720 (1994).

[21] W. Jaus, Phys. Rev. D41, 3394 (1990); 44, 2851 (1991).

[22] Demchuk et al., Phys. Atom. Nucl 59, 2152 (1996).

[23] G. Amoros, J. Bijnens and P. Talavera, Nucl. Phys. B 602, 87 (2001)

[24] O. Strandberg, arXiv:hep-ph/0302064.

[25] M. Knecht and A. Nyffeler, Eur. Phys. J. C 21, 659 (2001).

[26] J. Bijnens, Prog. Part. Nucl. Phys. 58, 521 (2007), and references therein. 